\title{
To Scan or Not to Scan: An Unnecessary Dilemma for PSMA Radioligand Therapy
}

\author{
Sandy Srinivas ${ }^{1}$ and Andrei Iagaru ${ }^{2}$ \\ ${ }^{1}$ Division of Medical Oncology, Department of Medicine, Stanford University, Stanford, California; and ${ }^{2}$ Division of Nuclear Medicine \\ and Molecular Imaging, Department of Radiology, Stanford University, Stanford, California
}

$\mathbf{T}$ he recently presented and published results of the VISION phase III study of ${ }^{177} \mathrm{Lu}-\mathrm{PSMA} 617$ for metastatic castrationresistant prostate cancer (1) are indeed one of the greatest success stories in the history of nuclear medicine (2). They add to the earlier experiences from first-in-humans studies (3-5) to phase II studies (6). However, danger lurks in this wave of unprecedented progress. The fact that more than $85 \%$ of screened participants met the ${ }^{68}$ Ga-PSMA11 eligibility criteria (1) made some question the need for prostate-specific membrane antigen (PSMA) PET imaging before ${ }^{177} \mathrm{Lu}$-PSMA treatment. This questioning of the need for PSMA PET is done without re-evaluating the eligibility criteria themselves (not all lesions had to be PSMA-positive) and their impact on study outcomes. Some will be ready to make the argument that excluding PSMA PET will eliminate costs and increase access to therapy. Let us next clearly make the case against this approach.

One of the undisputed principles of treating patients with cancer is knowing the extent of disease to the best of one's ability. Physicians practicing medical or radiation oncology who do not accurately define where cancer is and where it is not before using chemotherapy or external radiation would be viewed as derelict in their duties. So why would radioligand therapy for prostate cancer be treated any differently?

${ }^{177} \mathrm{Lu}$-PSMA617 is not expected have a low cost, nor is it without adverse events. Therefore, proper patient selection based on targeted PSMA PET imaging is necessary. By excluding patients unlikely to respond, PSMA PET will likely save health-care costs in the long run. It will also contribute prognostic factors for prediction of response to treatment (7), as well as reduce the possibility of adverse events by excluding ineligible patients. In fact, more PSMA PET imaging is likely needed during radioligand therapy, such as for evaluation of response to therapy mid-treatment and after the last cycle, replacing standard assessments with CT and bone scintigraphy.

The biology of prostate cancer is complex, and a single imaging test, no matter how good, may not be sufficient to properly select patients. The TheraP phase II study required baseline ${ }^{68} \mathrm{Ga}$-PSMA11 PET, as well as ${ }^{18} \mathrm{~F}$-FDG PET to exclude patients with active disease

Received Aug. 10, 2021; revision accepted Aug. 11, 2021.

For correspondence or reprints, contact Andrei lagaru (aiagaru@stanford. edu).

Published online August 26, 2021.

COPYRIGHT (C) 2021 by the Society of Nuclear Medicine and Molecular Imaging.

DOI: 10.2967/jnumed.121.263035 sites lacking PSMA expression (6). The outcomes, not surprisingly, appear superior to those reported in VISION and serve as a further argument for accurate eligibility screening.

Access to PSMA PET in the United States was a considerable issue before the Food and Drug Administration approval of 2 radiopharmaceuticals ( ${ }^{68} \mathrm{Ga}$-PSMA11 and ${ }^{18} \mathrm{~F}$-DCFPyL). With industry backing and logistics being addressed, the big limiting factor

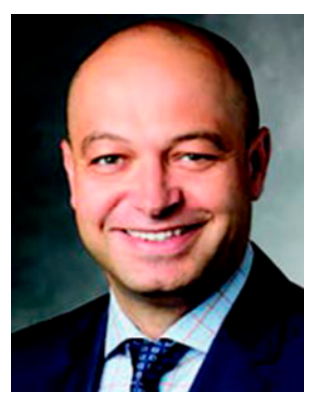

Andrei lagaru, MD will be inaccurate claims that imaging is not needed before treatment.

We have been through similar debates for the treatment of differentiated thyroid cancer (8). Some physicians still chose not to conduct a whole-body scan with a diagnostic activity of either ${ }^{131} \mathrm{I}$ or ${ }^{123}$ I. Others, including our group, always obtain this information. There are several reasons for this divergence of opinion. First, most patients with differentiated thyroid cancer have a very good prognosis. This is not the case with prostate cancer. Physicians often equate the excellent outcome with how they manage the cancer rather than with the natural history of the disease. They argue that if the patients have a good outcome without a whole-body scan then the scan is not necessary. Second, obtaining a posttherapy scan provides information regarding staging. But since when is it appropriate to stage disease after treating the patient? Third, stunning was debated and put to rest (9). There is no need to go through the same for radioligand therapy in prostate cancer.

European Association of Nuclear Medicine procedure guidelines for radionuclide therapy with ${ }^{177} \mathrm{Lu}$-labeled PSMA ligands properly include PSMA PET imaging for patient selection (10). These guidelines should be reviewed regularly and revised as needed to include emerging data demonstrating the benefits of PSMA PET imaging. Additional professional organizations should endorse them and make them a part of appropriate-use criteria.

The additional resources and cost to obtain the useful information from PSMA PET are a small fraction of what a prostate cancer patient and the health-care system will spend from the discovery of an elevated prostate-specific antigen level through referral to specialists, biopsy, surgery, radiation therapy, chemotherapy, hormonal therapy, radioligand therapy, and the long-term follow-up of the cancer. 
We are in the era of precision medicine and precision health (11), aiming to individualize therapies to achieve maximum benefit, including in prostate cancer. As an example, patients are getting genomic testing for BRCA mutations and would get a poly(adenosine diphosphate-ribose polymerase) inhibitor only if tested positive (12). Giving a patient such an inhibitor without testing is not accepted. We hope the prostate cancer medical community will stand up for precision medicine, including by ordering PSMA (and ${ }^{18}$ F-FDG) PET before treating a patient with ${ }^{177}$ Lu-PSMA617. PSMA radioligand therapy for prostate cancer without PSMA PET should not be accepted.

\section{DISCLOSURE}

No potential conflict of interest relevant to this article was reported.

\section{REFERENCES}

1. Sartor O, de Bono J, Chi KN, et al. Lutetium-177-PSMA-617 for metastatic castration-resistant prostate cancer. N Engl J Med. 2021;385:1091-1103.

2. Czernin J, Calais J. ${ }^{177}$ Lu-PSMA617 and the VISION trial: one of the greatest success stories in the history of nuclear medicine. J Nucl Med. 2021;62:10251026.
3. Delker A, Fendler WP, Kratochwil C, et al. Dosimetry for ${ }^{177}$ Lu-DKFZ-PSMA617: a new radiopharmaceutical for the treatment of metastatic prostate cancer. Eur J Nucl Med Mol Imaging. 2016;43:42-51.

4. Kratochwil C, Giesel FL, Stefanova M, et al. PSMA-targeted radionuclide therapy of metastatic castration-resistant prostate cancer with ${ }^{177}$ Lu-labeled PSMA-617. J Nucl Med. 2016;57:1170-1176.

5. Rahbar K, Ahmadzadehfar H, Kratochwil C, et al. German multicenter study investigating ${ }^{177} \mathrm{Lu}-\mathrm{PSMA}-617$ radioligand therapy in advanced prostate cancer patients. J Nucl Med. 2017;58:85-90.

6. Hofman MS, Emmett L, Sandhu S, et al. $\left[{ }^{177} \mathrm{Lu}\right]$ Lu-PSMA-617 versus cabazitaxel in patients with metastatic castration-resistant prostate cancer (TheraP): a randomised, open-label, phase 2 trial. Lancet. 2021;397:797-804.

7. Seifert R, Kessel K, Schlack K, et al. PSMA PET total tumor volume predicts outcome of patients with advanced prostate cancer receiving $\left[{ }^{177} \mathrm{Lu}\right] \mathrm{Lu}-\mathrm{PSMA}-617$ radioligand therapy in a bicentric analysis. Eur J Nucl Med Mol Imaging. 2021;48: 1200-1210.

8. McDougall IR. The case for obtaining a diagnostic whole-body scan prior to iodine 131 treatment of differentiated thyroid cancer. Thyroid. 2009;19:811-813.

9. McDougall IR, Iagaru A. Thyroid stunning: fact or fiction? Semin Nucl Med. 2011; 41:105-112.

10. Kratochwil C, Fendler WP, Eiber M, et al. EANM procedure guidelines for radionuclide therapy with ${ }^{177}$ Lu-labelled PSMA-ligands ( ${ }^{177}$ Lu-PSMA-RLT). Eur $J$ Nucl Med Mol Imaging. 2019;46:2536-2544.

11. Gambhir SS, Ge TJ, Vermesh O, Spitler R. Toward achieving precision health. Sci Transl Med. 2018; 10:eaao3612.

12. Anscher MS, Chang E, Gao X, et al. FDA approval summary: rucaparib for the treatment of patients with deleterious BRCA-mutated metastatic castrate-resistant prostate cancer. Oncologist. 2021;26:139-146. 